\title{
SIGDENGUE: Um Sistema de Informação para o Acompanhamento e Gestão de Ações sobre Dengue com Enfoque às Atividades de Notificação, Raio e Bloqueio
}

\author{
Claudia B. Rizzi, André L. Brun, Guilherme Galante, Rogério L. Rizzi \\ ${ }^{1}$ Centro de Ciências Exatas e Tecnológicas \\ Universidade Estadual do Oeste do Paraná \\ Cascavel-PR, Brasil. \\ \{claudia.rizzi, andre.brun, guilherme.galante, rogerio.rizzi\}@unioeste.br
}

\begin{abstract}
Dengue is an infectious disease caused by different virus serotypes whose main vector in Brazil is Aedes aegypti mosquito. Considering the lack of effective dengue vaccine, the option is to coordinate actions with the objective of controlling the vector proliferation. In Brazil, three information systems, SINAN, LIRAa and SISPNCD, are used for storing data on dengue. However, they are not integrated and are generally underutilized for governmental actions purposes. In this context, we present SIGDENGUE an information system for acquisition, manipulation and data treatment on Dengue. The practical use of the system shows that SIGDENGUE is an effective tool in for planning dengue prevention actions.
\end{abstract}

Resumo. A dengue é uma doença infecciosa causada por diferentes sorotipos de vírus e no Brasil, seu principal transmissor é o mosquito Aedes aegypti. Em decorrência da inexistência de vacina eficaz contra ela, ações são realizadas objetivando o controle do vetor. No país, o Sistema de Informação de Agravos de Notificação (SINAN), o Levantamento Rápido do Índice de Infestação por Aedes aegypti (LIRAa) e o Sistema do Programa Nacional de Controle da Dengue (SISPNCD) são os Sistemas de Informações mais utilizados para armazenamento de dados relativos à doença. Estes, no entanto, não estão integrados e geralmente são subutilizados para fins de gestão. É neste contexto que se insere o Sistema de Informação para Aquisição, Manipulação e Tratamento de Dados sobre a Dengue (SIGDENGUE). Suas principais funcionalidades são apresentadas bem como um experimento realizado em que se acompanhou as ações realizadas pelo Setor de Endemias do município de Cascavel/PR, desde a notificação do caso suspeito até o término das ações necessárias e decorrentes, utilizando-se o Sistema. Concluiu-se, dentre outros aspectos, que o SIGDENGUE foi efetivo no que diz respeito ao planejamento estratégico das ações realizadas bem como na agilização do trabalho de campo, fator decisivo na prevenção do espalhamento da doença. 


\section{Introdução}

A dengue é uma doença febril aguda causada por um arbovírus do gênero Flavivírus da família Flaviviridae. Diversas espécies de mosquitos Aedes podem servir como vetores transmissores da doença. No Brasil, duas delas estão instaladas, o Aedes albopictus e especialmente, o Aedes aegypti, um mosquito urbano, de hábito diurno e altamente ativo. A transmissão ocorre quando a fêmea da espécie se contamina ao picar um indivíduo infectado na fase de viremia, tornando-se capaz, após período de 10 a 14 dias, de transmitir o vírus a todos os indivíduos que picar [WHO 2006, WHO 2009].

Cada um dos sorotipos DEN-1, DEN-2, DEN-3, DEN-4 e mais recentemente DEN-5 [Mustafa et al. 2015], pode causar enfermidade grave e mortal e a infecção por um deles confere proteção permanente para o mesmo sorotipo e imunidade parcial e temporária contra os outros quatro. Mas a infecção subsequente com um segundo sorotipo aumenta a probabilidade de doença grave como a hemorrágica.

A dengue é característica de áreas tropicais e subtropicais e no Brasil, as condições socioambientais são favoráveis à expansão e dispersão do vetor. Desde a reintrodução da doença, em 1976, todos os anos são registradas novas ocorrências. O número de casos confirmados anualmente é bastante significativo, conforme se observa na Figura 1, referentes ao período de 1990-2014 [Portal da Saúde 2015].

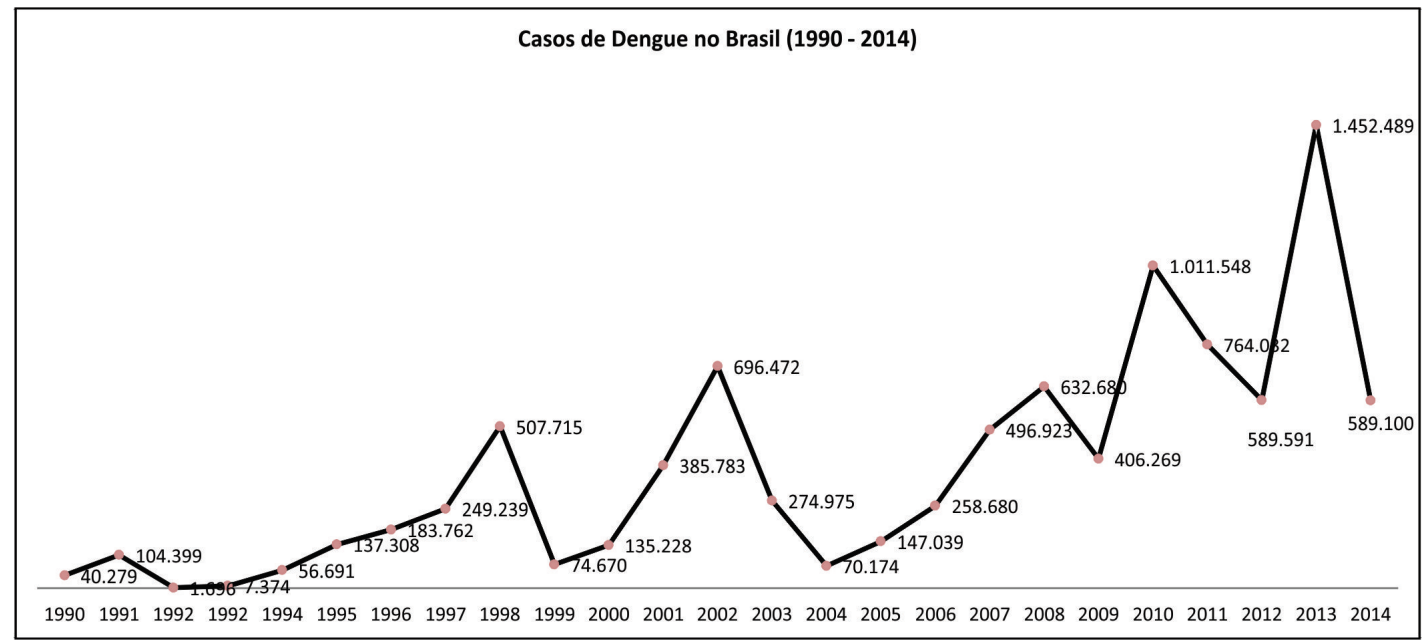

Figura 1. Casos de Dengue no Brasil, entre 1990 - 2014 [Portal da Saúde 2015]

Dados oficiais do governo brasileiro indicam que até setembro de 2015 estavam confirmados 1.438.497, sendo 1.318 casos de dengue grave e 17.183 casos de dengue com sinais de alarme. Foram confirmados 709 óbitos, o que representa um aumento no país de $71 \%$ em relação ao mesmo período de 2014. Além disso, em 18.281 amostras coletadas de indivíduos com suspeita de dengue realizou-se o exame de isolamento viral, das quais 7.381 foram positivas $(40,4 \%)$. Dessas, as proporções dos sorotipos virais identificados foram: DENV1 (93,4\%), seguido de DENV4 (5,4\%), DENV2 (0,8\%) e DENV3 (0,5\%) [Ministério da Saúde 2015a].

Esses dados fornecem subsídios para dimensionar o problema e em consequência, o trabalho que deve ser realizado em todo o país para minimizá-lo. Na ausência de vacina eficaz, a única ação para atuar na disseminação da doença é o controle do vetor. 
É neste contexto que se insere e se justifica o Sistema de Informações que está sendo desenvolvido no Laboratório de Computação Aplicada da UNIOESTE em parceria com a Prefeitura Municipal de Cascavel, Paraná. O sistema tem o objetivo de disponibilizar diversos componentes de software, incluindo georreferenciamento, que visam contribuir para a melhoria da saúde da população subsidiando ações de prevenção, controle e combate aos vetores da dengue. Embora tome a cidade de Cascavel como parceira e locus para o desenvolvimento de discussões teóricas e realização de experimentos, o sistema independe de região geográfica e pode ser utilizado por outros municípios, visto a metodologia e os recursos computacionais que oferece.

Este artigo está assim organizado. Na Seção 2 é feita uma revisão bibliográfica sobre sistemas computacionais em dengue nacionais e internacionais. Na Seção 3 as principais características do SIGDENGUE são apresentadas e é feita uma comparação com aquelas dos sistemas SINAN, LIRAa e SISPNCD. Na Seção 4 é feita uma síntese sobre a dinâmica do controle e combate à dengue realizadas por meio do SIGDENGUE em Cascavel e apresenta-se um exemplo do acompanhamento completo a um indivíduo suspeito de estar com dengue, compreendendo a notificação, a execução do Raio, Bloqueio e conclusão do caso via Sistema. Na Seção 5 são apresentados discussões a título de conclusão, bem como trabalhos em andamento.

\section{Sistemas Computacionais em Dengue}

Nesta seção são apresentados alguns dos Sistemas de Informação em dengue que foram identificados na revisão bibliográfica realizada. Inicialmente os Sistemas utilizados em âmbito nacional são sintetizados. Em seguida, uma visão mais abrangente é apresentada, incluindo alguns Sistemas internacionais e outros com objetivos específicos, mas relacionados à dengue.

\subsection{Sistemas Oficiais Brasileiros}

O Ministério da Saúde brasileiro realiza o controle e o monitoramento da dengue principalmente tendo como base informações obtidas por meio de três Sistemas de Informações: o Sistema de Informação de Agravos de Notificação (SINAN), o Levantamento Rápido do Índice de Infestação por Aedes aegypti (LIRAa) e do Sistema do Programa Nacional de Controle da Dengue (SISPNCD).

As informações contidas no SINAN referem-se, principalmente, à notificação e investigação de casos de doenças e agravos que constam na Lista Nacional de Doenças de Notificação Compulsória, que inclui a dengue. O objetivo principal do SINAN é realizar o registro e processamento dos dados, fornecendo informação para análise do perfil da doença, e contribuindo para a tomada de decisões em nível municipal, estadual e federal [Ministério da Saúde 2015c].

O LIRAa é realizado nas capitais e municípios de regiões metropolitanas, municípios com mais de 100 mil habitantes e municípios com grande fluxo de turistas ou situados em regiões de fronteira. O espaço geográfico é dividido em grupos de 9 a 12 mil imóveis com características semelhantes. Em cada grupo, são pesquisados 450 imóveis. Os grupos com índices de infestação predial inferiores a 1\% são considerados como estando em condições satisfatórias. Aqueles entre $1 \%$ a 3,9\% estão em situação de alerta. Considera-se que municípios com índices superiores a $4 \%$ há risco de surto de dengue [Ministério da Saúde 2015b]. 
O SISPNCD é o software por meio do qual são registradas as três principais ações executadas em campo pelos agentes que realizam o controle de endemias nos municípios brasileiros: o Ciclo, o Ponto Estratégico (PE) e a Pesquisa Vetorial Espacial (PVE). O Ciclo consiste em visitas bimestrais aos imóveis do município, visando à orientação a moradores, retirada, destruição ou limpeza de depósitos que podem acumular água e aplicação de larvicida. Os PEs são locais estratégicos, a exemplo de depósitos de lixos, cemitérios, borracharias, que, por suas características, podem contribuir para a proliferação do vetor. Esses locais devem ser vistoriados quinzenalmente com aplicação de larvicida e adulticida. O PVE é a ação realizada na circunvizinhança de 300 metros do local de maior permanência do indivíduo com suspeita de estar com dengue ou do local onde foram localizadas larvas ou pupas do vetor. Nesses locais é feita a remoção de depósitos e aplicação de larvicida [Ministério da Saúde 2010].

Ressalta-se que o preenchimento de dados nos três sistemas é obrigatório e que não há integração entre eles. Os sistemas apresentam várias limitações que vão desde a forma de inserção de dados, a manutenção de registros históricos, até o fornecimento de informações para o processo decisório. Atualmente, o uso destes sistemas resumese à produção de dados estatísticos nacionais. No âmbito municipal, os gestores responsáveis atuam principalmente considerando suas experiências sem o adequado suporte desses softwares.

\subsection{Outros Sistemas Voltados à Dengue}

No âmbito internacional, a importância do desenvolvimento de Sistemas de Informação Geográfica voltados para a vigilância da dengue com uso de tecnologias de acesso aberto tem ganho destaque, embora não seja recente [Duncombe et al. 2012]. O Grupo de Trabalho Científico sobre Pesquisa Dengue, convocado pelo Programa Especial para Pesquisa e Treinamento em Doenças Tropicais, realizado em 2006 em Genebra, já apontava que estes sistemas deveriam ser entendidos como uma nova geração de Sistemas de Informação com capacidade de gerenciar dimensões espaciais, em conjunto com noções temporais e relativas aos indivíduos envolvidos visando apoiar as ações de controle e combate à doença [Martinez 2006].

Anteriormente, mas contemporâneo a essas discussões, a Organização Mundial de Saúde desenvolveu em 2005 o DengueNet, um sistema de gerenciamento central de dados para a vigilância epidemiológica e virológica mundial da dengue baseado na Internet. Seu objetivo principal é disponibilizar uma plataforma padrão para o compartilhamento de dados visando detectar e monitorar a incidência e as tendências da dengue. O sistema viabiliza acesso a indicadores, como a incidência, letalidade, frequência e distribuição, a quantidade de mortes e os sorotipos circulantes. Os dados são apresentados graficamente, em mapas e formatos tabulares e objetivam fornecer informações que possam ser úteis a profissionais de saúde pública e gestores nas ações contra a doença. Estatísticas sobre a dengue podem ser acessadas a partir de 1955 e seu foco atual está na coleta global de dados [WHO 2015]. No entanto, há críticas quanto à confiabilidade e completeza dos dados que ali estão, considerando que se pretende usar uma escala mundial [Duncombe et al. 2012].

No âmbito nacional, em 2009, dez instituições brasileiras se organizaram em uma rede de pesquisa em torno da dengue, financiada pelo Conselho Nacional de Desenvolvimento Científico e Tecnológico (CNPq) por meio do Programa de Apoio a Núcleos e 
Excelência (PRONEX-Dengue) [Pronex-Dengue 2015]. As pesquisas realizadas por essa rede dedicaram-se em sua grande maioria, ao melhor entendimento da dengue, principalmente por meio de modelagem e simulação.

No contexto da modelagem e simulação, cabe destacar o DengueME. Elaborado pelo departamento de Computação da Universidade Federal de Ouro Preto com a participação de diversos pesquisadores, objetiva buscar padrões de transmissão do mosquito Aedes aegypti e determinar fatores ambientais, sociais e climáticos que contribuem para essa dinâmica em espaços geográficos reais. As simulações permitem a projeção de cenários e também avaliar a relação custo/benefício das diversas formas de controle da doença. Ele está sendo construído de maneira a ser de fácil manipulação para viabilizar que, além de pesquisadores acadêmicos, também profissionais de saúde possam realizar análises voltadas ao combate da dengue [TerraLab 2012]. Dispõe de dois módulos, o epidemiológico e o entomológico. O módulo epidemiológico atual contempla um modelo baseado em agentes que atuam em um espaço celular acoplado a um Sistema de Informações Geográficas, para simulação e análise de cenários da dinâmica espaço-temporal da difusão da dengue em ambientes urbanos reais. O módulo entomológico permite, por meio de dois modelos, simular o ciclo de vida do Aedes aegypti bem como as estratégias de controle da dengue que tem como alvo o vetor.

Nos encontros dos integrantes da rede Pronex-Dengue ocorridos em maio de 2013 e em novembro de $2014^{1}$, foram apresentados dois sistemas com o objetivo de subsidiar a gestão de ações práticas voltadas ao controle e combate à dengue: o Alerta-Dengue e o SIGDENGUE.

O Alerta Dengue, mais especificamente, o projeto denominado "Desenvolvimento de um sistema de alerta de surtos de dengue utilizando dados híbridos de redes sociais, monitoramento entomológico, epidemiológico e climático" é coordenado pela Fundação Getúlio Vargas e Fundação Oswaldo Cruz. Eles desenvolveram um Sistema capaz de emitir "alertas de dengue" considerando dados climáticos (temperatura), epidemiológicos (notificação de dengue) e do twitter. Poderá também considerar dados entomológicos (LIRAa e ovitrampas). Trata-se de um Sistema cuja participação está aberta aos municípios interessados que a ele poderão se agregar fornecendo os referidos dados. A expectativa é a de que seja possível ampliar o conhecimento sobre a disseminação e o controle da dengue em âmbito nacional.

Em funcionamento para o município do Rio de Janeiro, o Alerta Dengue é denominado InfoDengueRio [PROCC 2015]. Os dados são disponibilizados pela secretaria de saúde do município na forma de relatórios semanais, com a quantidade de casos notificados de dengue por bairro. Dados relativos a menção à dengue via Twitter e dados climáticos são obtidos automaticamente por meio do sistema permitindo indicar eventos numa velocidade mais rápida do que as notificações clássicas. Por esta característica, a expectativa é a de que se possa agilizar o processo de tomada de decisão na vigilância da dengue visando permitir rápido acesso a diagnósticos semanais de situação e prognósticos de curta duração. Busca-se atender a necessidade de gerar informações em tempo quasereal para tomada de decisão. Isto porque, o controle da incidência e preparação para o enfrentamento de epidemias de dengue dependem de um monitoramento de sinais que

${ }^{1}$ http://www. dengue.mat.br/stories/eventos.html 
pressagiam o aumento de casos e potenciais epidemias. Para isso, são utilizadas metodologias estatísticas robustas capazes de produzir alertas epidemiológicos subsidiando uma ação eficaz, oportuna e específica.

Foco deste trabalho, o SIGDENGUE é apresentado em detalhes nas próximas seções. Dotado de funcionalidades para fins de controle e combate à dengue, o Sistema contempla as questões metodológicas e funcionais preconizadas pelo Ministério da Saúde e implementa atividades e ações operacionais na perspectiva municipal, ausentes em outros Sistemas pesquisados. Adicionalmente, estão sendo realizadas discussões sobre sua integração com o Alerta Dengue visto que pode prover os dados por ele utilizado.

Há outros Sistemas computacionais nacionais com enfoque semelhante em certos aspectos. Alguns referem-se a projetos que foram iniciados e descontinuados. Outros, apresentam características específicas, voltadas para municípios específicos. Exemplos desses sistemas são citados a seguir.

O Focos Online é um software baseado na Internet, desenvolvido pela Secretaria de Estado da Saúde do estado do Tocantins que permite a identificação em tempo real, da localização por ruas, quadras e bairros dos locais que podem ser focos de proliferação do Aedes aegypti. Permite a inclusão de imagens desses imóveis e disponibiliza um modelo de notificação para os proprietários que serve como instrumento de apoio para ações dos agentes de campo [Sesau-TO 2015].

Com abordagem semelhante, o Observatório Dengue vinculado ao projeto Telessaúde, é um projeto voltado ao estado do Rio Grande do Norte. A proposta original era de que as informações decorrentes das visitas realizadas pelos agentes de campo fossem informadas por meio de dispositivos móveis, em tempo real no âmbito estadual, permitindo que o cidadão pudesse ter acesso e acompanhasse o trabalho realizado [Barros et al. 2013]. Atualmente ele disponibiliza formulário para que o cidadão possa indicar um possível foco de mosquito, objetivando a rápida ação das equipes.

Restrito à Salvador-BA, o Dengue na Web visa identificar os locais onde ocorrem os casos da doença na cidade de Salvador, buscando o envolvimento e a participação dos cidadãos. De posse de um endereço eletrônico e efetuado o registro no site, o indivíduo pode informar seus sintomas, caso suspeite estar com a doença ou caso tenha informações sobre a ocorrência de casos na cidade. Uma vez cadastrado no site, os participantes recebem notícias a respeito da dengue e são convidados a responder um questionário sobre os sintomas observados na semana. Esses dados são utilizados para acompanhar, em tempo real, a evolução da doença em um período de tempo por meio de dados estatísticos, mapas georreferenciados, dentre outros, que ficam disponíveis no site. Paralelamente estão sendo desenvolvidos modelos matemáticos para simular a propagação da doença em Salvador objetivando desenvolver cenários de intervenção [Instituto de Saúde Coletiva - UFBA 2015].

Com uma abordagem comercial, serviços relativos ao trabalho com Dengue são oferecidos pela empresa Ecovec [Ecovec 2015]. Por exemplo, por meio do MI-Dengue são associadas armadilhas desenvolvidas para captura de mosquitos Aedes que são distribuídas em uma área urbana. Essas armadilhas são vistoriadas pelos agentes de endemias que informam à central, por meio de aparelhos celulares, a quantidade de capturas em cada uma delas. Esses dados são processados e disponibilizados em um site 
específico que georreferencia os locais de infestação do vetor. Apresenta também tabelas de incidência em áreas específicas como bairros e fornece relatórios e informações para a condução de trabalhos de campo realizado pelos agentes de endemias.

Com uma proposta que se assemelha em alguns aspectos ao SIGDENGUE, foi desenvolvido um Sistema de Informações para o município de Campinas, São Paulo, visando o controle da dengue utilizando geoprocessamento. Foram georreferenciados e analisados casos ocorridos em 2001 e 2002 e foi iniciada sua utilização em ações de rotina nos anos de 2002 e 2003 [Lima et al. 2004]. Também trabalharam usando mapas para facilitar as atividades de planejamento e controle da dengue.

\section{SIGDENGUE}

O Sistema de Informação para Aquisição, Manipulação e Tratamento de Dados sobre a Dengue (SIGDENGUE) possui dois objetivos principais. O primeiro é integrar os dados e informações sobre dengue que o município de Cascavel dispunha e que não estavam armazenados e acessíveis em meio digital apropriado, bem como sobre outros dados relacionados à doença, como o acompanhamento dos casos suspeitos e confirmados, informações sobre pontos estratégicos, localidades, resultados laboratoriais decorrentes de material colhido em visitas a campo, equipes de trabalho, dentre outras situações, incluindo a integração de algumas informações armazenadas nos sistemas computacionais SINAN, LIRAa e SISPNCD. Todos esses dados eram acompanhados manualmente ou por meio de arquivos impressos e a cada ano eram arquivados, descontinuados ou descartados. O segundo objetivo é oportunizar a rápida e confiável obtenção de informações por meio de consultas e relatórios sobre dengue e a visualização em mapas georreferenciados, visando subsidiar a gestão das ações operacionais.

A sistemática quanto à operacionalização das ações e o respectivo registro de dados foram desenvolvidos e aperfeiçoados em conjunto o Setor de Endemias de Cascavel. Contempla não apenas a identificação dos casos suspeitos de dengue mas outras informações que ampliam e reforçam as possibilidades de ação dos gestores que podem atuar intervindo nos locais em que há maior probabilidade de que o vírus se espalhe.

Principalmente este aspecto, o do registro digital das informações geradas pelo trabalho realizado em campo, motivou a equipe da Universidade Estadual do Oeste do Paraná (Unioeste) a priorizar o desenvolvimento do SIGDENGUE para atender especificamente esta demanda, visto que atua também com modelagem e simulação. Evidenciouse que, de posse dos dados registrados, seria possível obter informações fundamentais, muitas vezes custosas ou impossíveis de serem elaboradas manualmente em tempo hábil para apoiar a gestão e consequentemente o direcionamento das ações em campo.

A primeira versão do SIGDENGUE foi implantada no Setor de Endemias de Cascavel, em 2012. Tratava-se de uma versão desktop e suas principais funcionalidades eram: Acompanhamento de indivíduos com suspeita de estarem com a dengue; Gerenciamento do Raio; Gerenciamento do Bloqueio; Gerenciamento de Pontos Estratégicos; Gerenciamento de Amostras Laboratoriais; Acompanhamento do LIRAa; Acompanhamento do Mutirão; Armazenamento dos dados provenientes do Sistema de informações de Febre Amarela e Dengue (SISFAD) substituído no final de 2012, pelo SISPNCD; Geração de Relatórios que permitiam o monitoramento e controle do risco da transmissão da dengue e o acompanhamento de estratégias gerenciais sobre a dengue. 
Na Tabela 1 apresentam-se as principais características dos softwares oficiais utilizados no registro e controle da dengue no Brasil (seção 2.1) e uma comparação com o SIGDENGUE.

Tabela 1. Características dos software de controle de Dengue

\begin{tabular}{|c|c|c|c|c|}
\hline Característica & SIGDENGUE & SINAN & LIRAa & SISPNCD \\
\hline Dados sobre pontos estratégicos & $\checkmark$ & & & $\checkmark$ \\
\hline Dados sobre localidades & $\checkmark$ & & & $\checkmark$ \\
\hline Dados sobre larvas e pupas & $\checkmark$ & & & $\checkmark$ \\
\hline Dados sobre controle mecânico & $\checkmark$ & & & $\checkmark$ \\
\hline Dados sobre controle químico & $\checkmark$ & & & $\checkmark$ \\
\hline Levantamento Amostral Instantâneo & $\checkmark$ & & $\checkmark$ & \\
\hline $\begin{array}{l}\text { Detalhamento de indivíduos com sus- } \\
\text { peita de dengue }\end{array}$ & $\checkmark$ & $\checkmark$ & & \\
\hline $\begin{array}{l}\text { Identificação georreferenciada de in- } \\
\text { divíduos com suspeita de dengue }\end{array}$ & $\checkmark$ & & & \\
\hline $\begin{array}{l}\text { Acompanhamento do Raio associado } \\
\text { ao caso suspeito }\end{array}$ & $\checkmark$ & & & \\
\hline $\begin{array}{l}\text { Acompanhamento do Bloqueio associ- } \\
\text { ado ao caso confirmado }\end{array}$ & $\checkmark$ & & & \\
\hline $\begin{array}{l}\text { Acompanhamento dos casos suspeitos } \\
\text { e confirmados de dengue }\end{array}$ & $\checkmark$ & & & \\
\hline Cadastro das equipes de campo & $\checkmark$ & & & $\checkmark$ \\
\hline $\begin{array}{l}\text { Dados sobre trabalho realizado pelas } \\
\text { equipes de campo }\end{array}$ & $\checkmark$ & & & $\checkmark$ \\
\hline Outras ações em dengue, mutirões & $\checkmark$ & & & \\
\hline Dados históricos & $\checkmark$ & & & $\checkmark$ \\
\hline Apoio na tomada de decisão & $\checkmark$ & & & $\checkmark$ \\
\hline
\end{tabular}

Pelas características apresentadas na Tabela 1, nota-se que o SIGDENGUE é um Sistema de Informações peculiar no sentido de que reúne dados e produz informações sobre a dengue no âmbito da administração municipal e contribui para a deflagração de ações pertinentes e embasadas. O principal diferencial do SIGDENGUE reside no fato de que foi idealizado considerando a dinâmica do trabalho realizado em campo, tendo como elemento norteador das ações, os casos de indivíduos com suspeita de estarem com dengue.

A síntese do processo de acompanhamento desses casos, que compõe o conjunto de testes operacionais com o sistema é apresentada nas próximas seções. É feita com uma abordagem qualitativa, enfocado o ponto de vista dos diversos usuários do Sistema, sejam agentes de saúde que atuam no campo, supervisores de equipes ou mesmo o responsável pelo Setor de Endemias. O objetivo é relatar a dinâmica do processo e realizar as análises pertinentes considerando as ações práticas e os registros no SIGDENGUE.

A metodologia utilizada para o desenvolvimento do projeto SIGDENGUE contempla as subdivisões estruturadas operacionalmente objetivando otimizar as ações da equipe que nele atua. De modo abrangente, para o desenvolvimento da nova versão do Sistema estão sendo seguidas as especificidades da Engenharia de Software empregando 
a metodologia de desenvolvimento de software Feature-Driven Development (FDD) [Palmer and Felsing 2002], e da Geomática para aquisição, tratamento e disponibilização dos dados [Azevedo 2007]. O detalhamento dos aspectos relacionados ao projeto e desenvolvimento do sistema estão fora do escopo pretendido para este trabalho.

\section{SIGDENGUE em Uso: a Dinâmica do Controle e Combate à Dengue}

Para um indivíduo que procura assistência médica apresentando sintomas da dengue, é obrigatório por força de lei (Portaria $n^{\circ}$. 104/2011 [Ministério da Saúde 2011]), o preenchimento da "Ficha de Investigação de Dengue" por parte do estabelecimento de saúde que o atendeu que também o encaminha para realizar o exame laboratorial para confirmar ou não a doença. Os dados deste formulário são cadastrados no software SINAN e uma cópia é encaminhada para o Setor de Endemias do município. O Setor entra em contato com este indivíduo ou com seus familiares para obter outras informações sobre a evolução do quadro e preenche um formulário cujos dados são digitados no SIGDENGUE. O preenchimento da "Ficha de Investigação de Dengue" e o complemento com outras informações sobre o paciente e seu quadro clínico constitui a ação de Notificação.

Mesmo sem a confirmação de que se trata de um caso positivo de dengue, o Setor de Endemias executa o procedimento de campo denominado "pesquisa espacial do vetor" ou simplesmente Raio. Neste procedimento são eliminados criadouros e são recolhidas amostras de larvas e pupas encontradas em um raio de 300 metros de um ou mais endereços mais frequentados pelo indivíduo, como sua residência, local de trabalho, estudo ou lazer. As informações decorrentes desta ação de campo são cadastradas no SISPNCD e no SIGDENGUE.

As amostras recolhidas durante o procedimento do Raio são encaminhadas para análise laboratorial e caso sejam positivas, é realizada a atividade denominada "combate local ao vetor alado" ou simplesmente Bloqueio. Trata-se da aplicação de inseticida de ação residual visando atingir o mosquito adulto na tentativa de impedir que o vírus se propague. Concluído o Bloqueio, ou caso a análise laboratorial das amostras de larvas e pupas seja negativa, o trabalho realizado pelo Setor de Endemias é considerado concluído. O fluxo da dinâmica do trabalho realizado para cada caso suspeito de dengue é ilustrado pela Figura 2.

Independentemente dos casos suspeitos de dengue, ao longo do ano a equipe de campo realiza atividades sistemáticas de visitas aos imóveis da cidade para a realização de ações de educação e de prevenção junto a moradores, o que inclui os levantamento de índice de infestação e o tratamento, também chamada de ação LI+T. Trata-se da ação realizada pelo agente que, ao visitar o imóvel, seja terreno baldio, ponto estratégico, residência ou imóvel comercial, inspeciona depósitos de água que constituem criadouros potenciais. Exemplos são caixas de água, pneus, pequenos depósitos que armazenam água, lixo, depósitos naturais, etc. Esses depósitos são inspecionados e alguns são eliminados. Quando um endereço que corresponde a um local de frequência de um indivíduo suspeito de estar com dengue é notificado, a ação de Raio não é realizada se as ações de LI+T foram executadas em um período de 15 dias da data de notificação do caso suspeito.

Embora o SIGDENGUE viabilize outros componentes de software, conforme ilustrado na Tabela 1, a ênfase neste trabalho é dada às ações de Notificação, Raio e Bloqueio. Esta opção se justifica visto sua importância na prevenção, controle e combate 


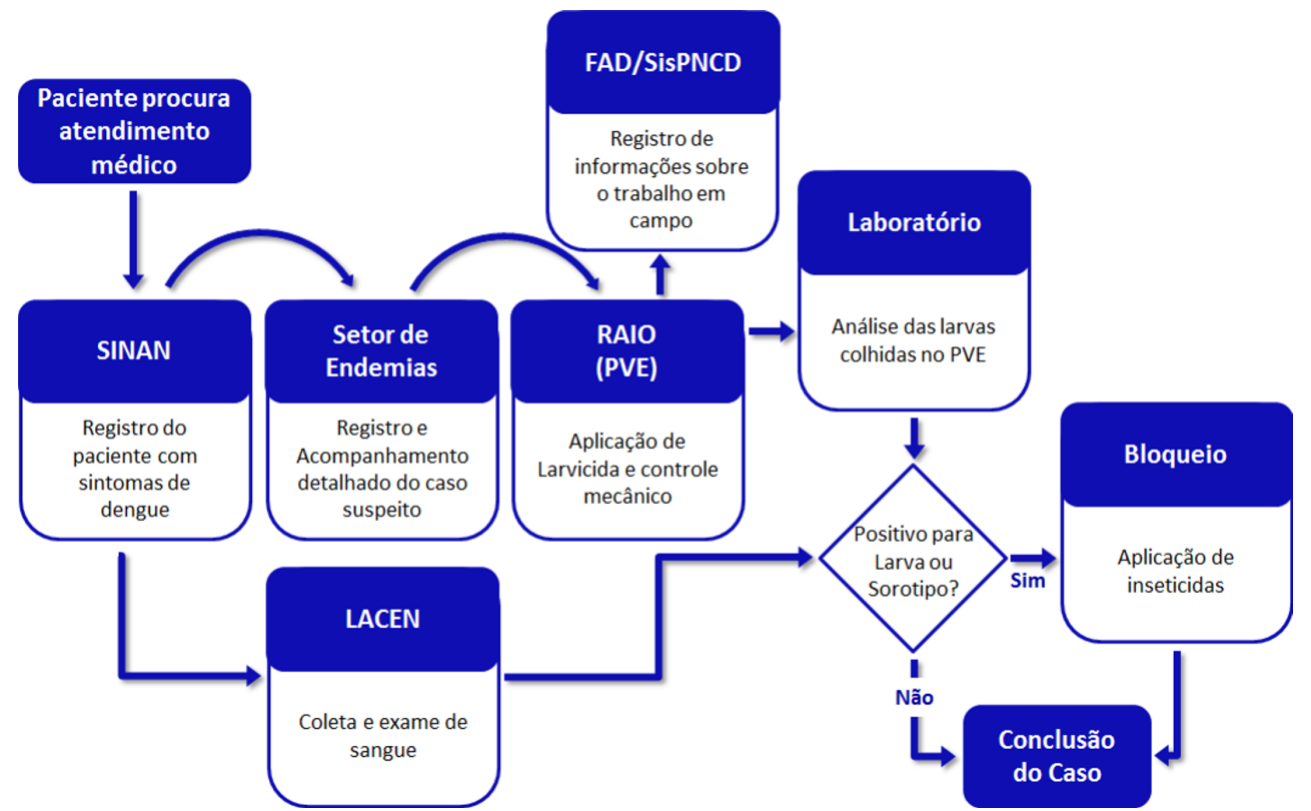

Figura 2. Síntese do fluxo da dinâmica do trabalho realizado para cada caso suspeito de dengue realizado pelo Setor de Endemias de Cascavel - PR.

à doença e também às principais características que constituem o diferencial do sistema. Para ilustrar a dinâmica que o SIGDENGUE viabiliza, essas ações são exemplificadas por meio da apresentação de um caso real em que um indivíduo, identificado como Paciente $\mathrm{X}$, contraiu dengue em janeiro de 2013.

\subsection{Exemplo de Notificação, Raio e Bloqueio no SIGDENGUE}

No dia 17/01/2013, apresentando sintomas associados à dengue, o Paciente $\mathrm{X}$ procurou um hospital da cidade que preencheu a "Ficha de Investigação de Dengue". De posse desta ficha, o Setor de Endemias entrou em contato telefônico com familiar do paciente solicitando informações pessoais, clínicas, sintomas, medicamentos, exames, se viajou ou não e os locais de sua frequência, que são categorizados no SIGDENGUE como residência, estudo, trabalho, lazer e outros. As Figuras 3(A), (B) e (C) ilustram 3 dos 12 formulários disponíveis para o registro e acompanhamento de cada caso suspeito de dengue e a Figura 3(D) apresenta um dos formulários de atividades realizadas na ação Raio visando o respectivo acompanhamento do trabalho de campo realizado.

Analisando o caso do Paciente X, profissionais de Saúde do Setor de Endemias consideram seus sintomas (Figura 3(C)), a data dos primeiros sintomas, medicamentos utilizados (Figura 3(B)) e os locais onde permanecia na maior parte do tempo, verificando se nesses locais havia notificação recente de outros casos da doença. Destaca-se na Figura 3(D) o local de sua residência (bairro Brasília) e de seu trabalho (bairro Pacaembu). Embora tenham classificado o caso como gravíssimo do ponto de vista de controle do vetor, identificaram que no local de sua residência haviam sido realizadas ações de LI+T em um período inferior a 15 dias. Ou seja, todo o trabalho de LI+T realizado no bairro Pacaembu, próximo ao endereço de trabalho do indivíduo se deu no período entre os dias 6/01/2013 e 12/01/2013. Sendo assim, a ação de Raio respectiva ao local de trabalho do caso suspeito não foi realizada. 


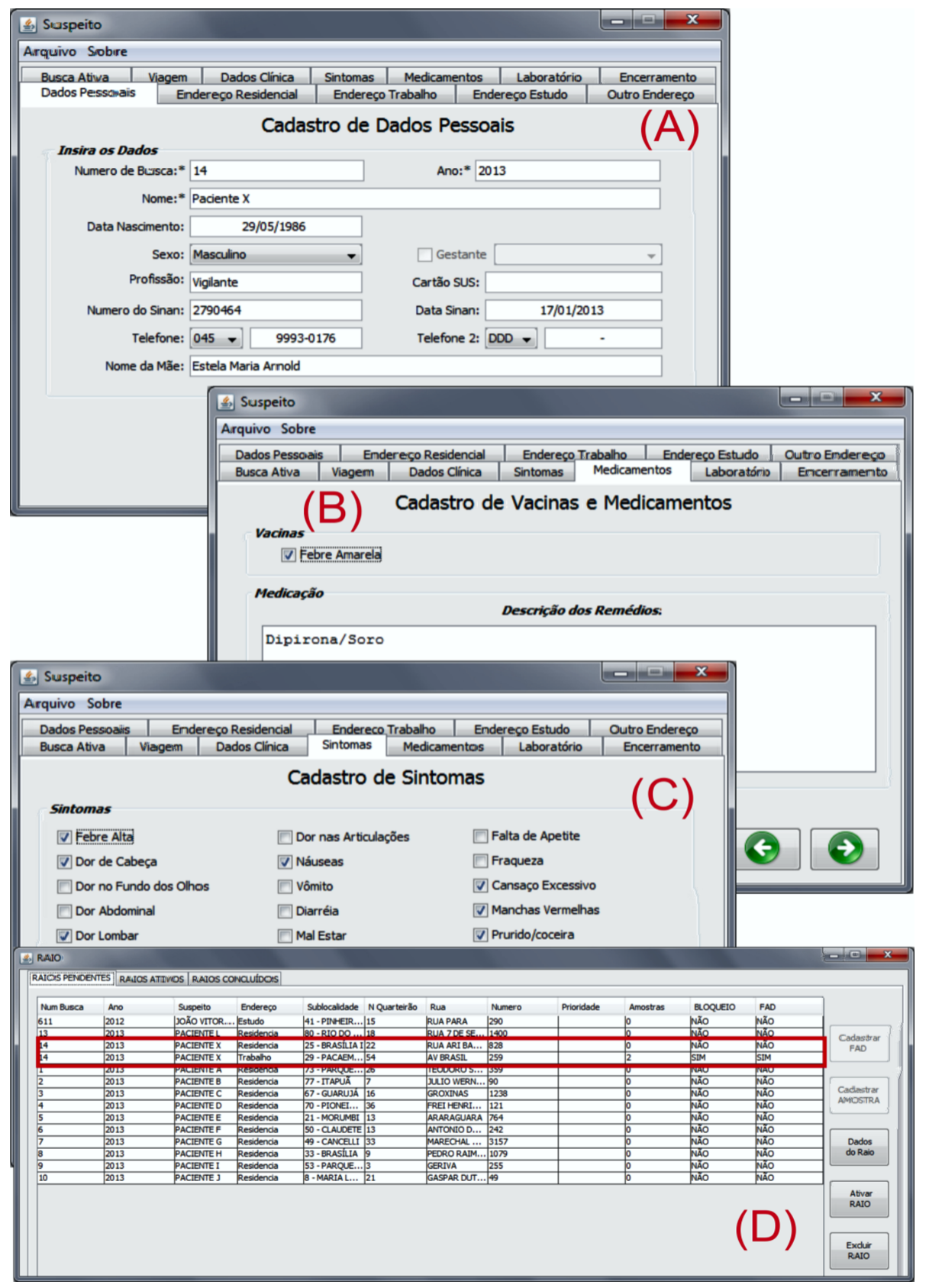

Figura 3. Ilustração de 4 telas do SIGDENGUE: (A) Notificação do caso de suspeita de dengue do Paciente $X$; (B) relação de vacinas e medicamentos; (C) Identificação de seus sintomas; (D) Destaque dos Raios efetuados referentes ao Paciente $X$.

$\mathrm{Na}$ ação de LI+T correspondente foram inspecionados 70 imóveis sendo 45 residências, 13 comércios, 10 terrenos baldios e 2 outros imóveis. Ao todo, 16 imóveis não foram visitados, visto que estavam fechados. Foram inspecionados 162 depósitos dos seguintes tipos: 4 depósitos de armazenamento de água, 20 pequenos depósitos móveis, 4 depósitos de lixo, 125 recipientes plásticos e sucatas, e 9 depósitos naturais. Foram eliminados 190 recipientes. Sete deles foram tratados com larvicidas. Foram colhidas 5 amostras que foram analisadas em laboratório. Dessas, 38 eram larvas e 13 pupas. Uma delas era do Aedes aegypti. Este fato indicou para os gestores do Setor de Endemias 
que aquela região apresentava alto risco na proliferação da dengue: além do indivíduo com suspeita da doença trabalhar naquele bairro, uma das amostras colhidas no trabalho corriqueiro de controle da doença foi positiva para o mosquito.

A ação de Raio no local de residência desse indivíduo foi realizada no dia 25/01/2013 e nela inspecionaram-se 117 imóveis sendo 95 residências, 9 comércios, 11 terrenos baldios e 2 outros imóveis. Ao todo, 60 imóveis não foram visitados porque estavam fechados. Foram inspecionados 148 depósitos dos seguintes tipos: 6 depósitos de armazenamento de água, 39 pequenos depósitos móveis, 102 recipientes plásticos e sucatas, e 1 pneu. Foram eliminados 108 recipientes, sendo que 4 foram tratados com larvicidas. Foram colhidas 4 amostras que foram analisadas em laboratório cujo resultado foi divulgado no dia 27/01/2013. Sete eram larvas e 9 pupas. Quatro delas eram do Aedes aegypti.

Neste caso, procedeu-se a realização do Bloqueio nas proximidades de seu local de trabalho e também residência. Esta decisão baseou-se na presença do Aedes aegypti em amostras obtidas na ação LI+T próxima ao local de trabalho bem como próxima ao local de residência do suspeito. Além disso, paralelamente, o paciente submeteu-se a exame laboratorial realizado no Laboratório Central do Estado do Paraná, que colheu a amostra no dia 18/01/2013 e divulgou o resultado no dia 23/01/2013, confirmando que o Paciente $\mathrm{X}$ estava com dengue.

Para efeitos de distribuição e operacionalização das diversas atividades de campo realizadas pelas equipes de agentes no controle ao vetor, o Setor de Endemias do $\mathrm{Mu}-$ nicípio sob orientação do Ministério da Saúde subdividiu a região sob sua responsabilidade em localidades. A Figura 4 ilustra a área urbana da cidade de Cascavel dividida nessas localidades. Na figura, são destacadas suas sub-regiões, que designam as vizinhanças dos locais da residência e do trabalho do Paciente $X$, mostrando a abrangência das ações de Raio e Bloqueio realizados. Concluído o Bloqueio, procedeu-se o encerramento do acompanhamento do caso do Paciente X.

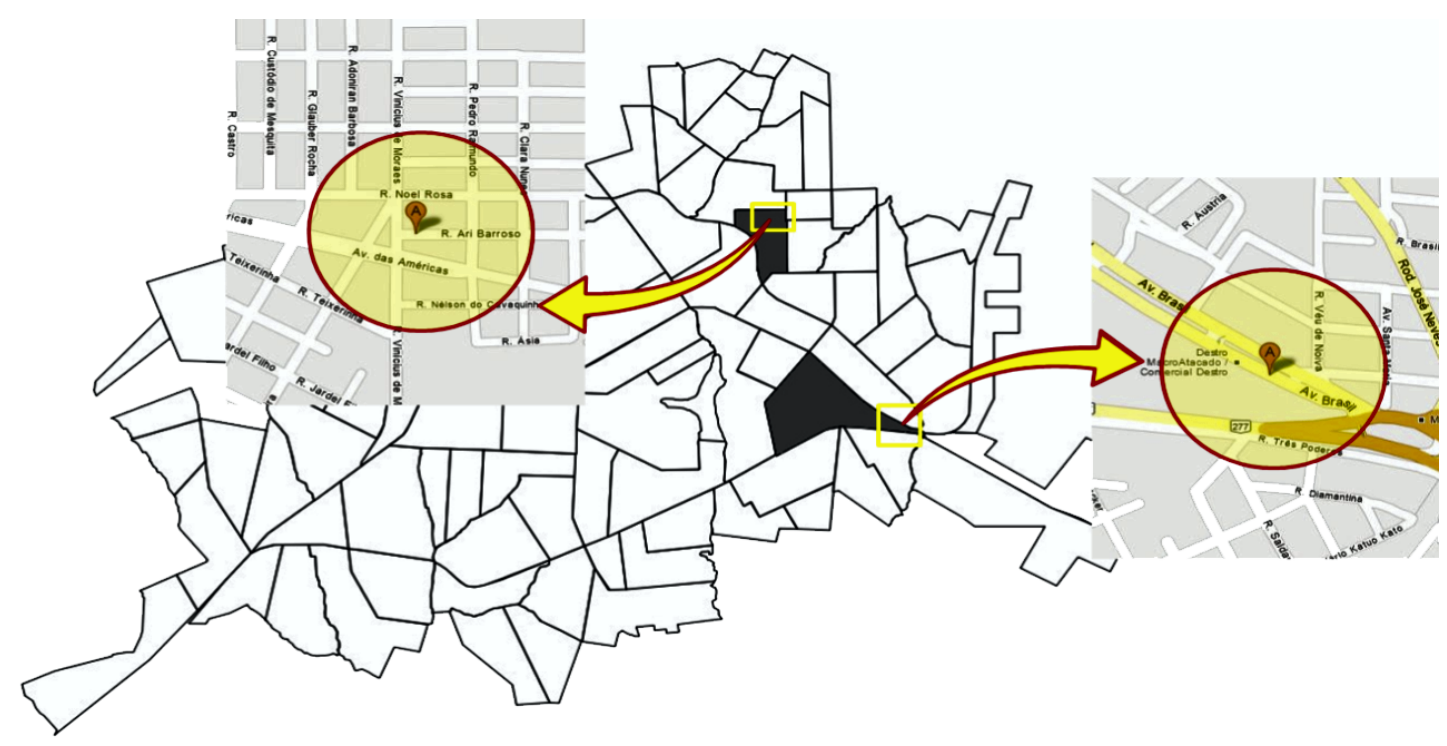

Figura 4. llustração das Localidades de Dengue no Município e os dois locais de Raio e do Bloqueio realizados em decorrência do Paciente X. 


\subsection{Tomada de Decisões em Dengue}

O SIGDENGUE implementa o registro de diversas informações incluindo aquelas constantes nos principais softwares brasileiros oficiais (SINAN, LIRAa e SISPNCD), além de viabilizar a geração de informações que subsidiam melhores condições à tomada de decisão e a organização e acompanhamento da dinâmica do trabalho realizado pelos agentes em campo. No caso do Paciente $X$, o processo que envolveu sua notificação, o trabalho decorrente e o encerramento do caso foi realizado no período de 15 dias, conforme a Tabela 2 mostrada a seguir.

Tabela 2. Características dos software de controle de Dengue

\begin{tabular}{lll}
\hline Ação & Data & Desdobramentos \\
\hline Notificação do caso & $17 / 01 / 2013$ & $\begin{array}{l}\text { No mesmo dia, um agende de saúde entrou em } \\
\text { contato telefônico com a família do Paciente X e } \\
\text { obteve outras informações sobre o caso. }\end{array}$ \\
\hline Exame laboratorial & $18 / 01 / 2013$ & $\begin{array}{l}\text { O Paciente X foi orientado e realizou o exame de } \\
\text { dengue no laboratório credenciado. }\end{array}$ \\
\hline Realização do Raio & $25 / 01 / 2013$ & $\begin{array}{l}\text { Realizada ação de eliminação de criadouros e co- } \\
\text { leta de amostras de larvas e pupas. }\end{array}$ \\
\hline $\begin{array}{l}\text { Conclusão da análise la- } \\
\text { boratorial }\end{array}$ & $27 / 01 / 2013$ & $\begin{array}{l}\text { Confirmada a presença de formas imaturas do Ae- } \\
\text { des aegypti próxima a casa do Paciente X. } \\
\text { Decisão pela realização do Bloqueio. }\end{array}$ \\
\hline Realização do Bloqueio & $28 / 01 / 2013$ & $\begin{array}{l}\text { Realizada ação de aplicação de inseticida na área } \\
\text { próxima à residência do Paciente X. }\end{array}$ \\
\hline Realização do Bloqueio & $31 / 01 / 2013$ & $\begin{array}{l}\text { Realizada ação de aplicação de inseticida na área } \\
\text { próxima ao local de trabalho do Paciente X. }\end{array}$ \\
\hline Conclusão do caso & $31 / 01 / 2013$ & $\begin{array}{l}\text { Encerramento do caso e cômputo como mais um } \\
\text { caso positivo em Cascavel - PR. }\end{array}$ \\
\hline
\end{tabular}

Embora o Setor de Endemias julgue o caso do Paciente X como um caso típico que recebeu um atendido considerado adequado, diversas são as análises que eles fazem com relação ao processo. Consideram que o uso do SIGDENGUE viabilizou e agilizou o acompanhamento de cada caso suspeito. Até então, todo o processo sintetizado na Tabela 2 era feito em fichas com anotações manuais, distribuídas em locais físicos (pastas) diferentes. Levando em consideração a necessidade de acompanhamento simultâneo de diversos casos notificados, que caracteriza o trabalho diário naquele Setor, os gestores não dispunham de condições propícias para planejar e executar as ações necessárias. Com o SIGDENGUE, podem, por meio de relatórios ou simples consultas ao sistema, comparar os casos, considerar o local de maior frequência do indivíduo com suspeita de estar contaminado, priorizar determinadas ações e melhor acompanhar o trabalho realizado pela equipe. Esta possibilidade tem alterado a forma de atuação em campo conferindo a ela mais qualidade e agilidade.

Outra análise realizada diz respeito ao fato de que o Setor de Endemias já suspeitava de forma empírica que no trabalho realizado por eles havia uma distância temporal significativa entre o momento da notificação do caso até sua conclusão. Por meio do SIGDENGUE, com o registro de cada ação realizada, tem sido possível acompanhar cada caso individualmente e analisar os motivos para maior ou menor demora na deflagração 
das ações. No exemplo do Paciente X, foram 15 dias de trabalho. Reconhece-se que o tempo necessário para a execução desse processo precisa ser minimizado, mesmo considerando que o trabalho realizado em campo depende das condições climáticas. No período em questão (entre 17 e 31/01/2013), o tempo estava estável, com temperaturas médias variando entre 18 e $31^{\circ} \mathrm{C}$ e não houve chuva em Cascavel. Nessas condições, e levando em conta a sazonalidade da dengue no município (janeiro é um mês considerado de risco), a celeridade das ações realizadas pelo Setor carece de melhorias significativas.

O rápido e eficaz gerenciamento é uma exigência que implica na quantidade do trabalho determinada também pela equipe responsável pelas ações realizadas. Essas ações devem ocorrer mesmo em situações adversas como condições climáticas e ambientais e depende de decisões técnicas e administrativas que, se não corretas e ágeis, acarretaram em atraso inoportuno numa necessidade de atuação emergencial. Da ação ágil e objetiva pode ser possível evitar a proliferação do vírus, bem como os riscos que potencializam o aumento na quantidade de indivíduos doentes. Essas e outras questões evidenciam a necessidade de contínuo e intenso trabalho de planejamento, treinamento e mobilidade das equipes e das ações realizadas, e o SIGDENGUE tem se mostrado uma ferramenta importante de apoio as atividades realizadas.

Sob a ótica macroambiental, cabem alguns comentários. As metodologias preconizadas pelo Ministério da Saúde, órgão federal brasileiro máximo que determina e companha as ações implementadas pelos municípios, visam o efetivo controle da doença. No entanto, diversos são os fatores que influenciam no alcance desta meta. Compreendem desde concepções políticas, prioridades e disponibilidade de verbas, desenvolvimento e utilização de sistemas de informações locais (como o SIGDENGUE) dentre outros. Há também as características ambientais próprias regionais bem como o dinamismo da transmissão da doença. Essas questões fazem com que municípios aparentemente semelhantes alcancem resultados distintos. Além disso, as metodologias federais e as concretizadas localmente podem ser conduzidas de formas diferentes, podendo levar a cenários não complementares ou coordenados. Podem ser direcionadas ao alcance de metas puramente estatísticas fazendo com que seus resultados possam não representar qualitativamente a realidade local, nem subsidiar adequadamente a tomada de decisões.

\section{Conclusão e Trabalhos Futuros}

Os Sistema de Informação (SI) são mecanismos de coleta, processamento, análise e produção de informações necessárias para planejar, organizar, executar e avaliar ações e serviços. As informações geradas pelos SIs devem contribuir para reduzir incertezas, identificar prioridades, auxiliar no planejamento e na execução de ações que promovam os resultados necessários e esperados.

No caso específico do SIGDENGUE, suas funcionalidades podem efetivamente apoiar o trabalho gerencial no que se refere ao controle e combate à dengue. Como mostrado nas Seções 4.1 e 4.2, as informações disponibilizadas pelo sistema permitem que decisões importantes relacionadas ao controle da doença possam ser tomadas pelos órgãos responsáveis de modo efetivo e rápido. Isso só é possível no SIGDENGUE devido todas as informações estarem centralizadas e disponíveis para os gestores, diferentemente no que ocorre nos demais sistemas voltados ao controle da dengue.

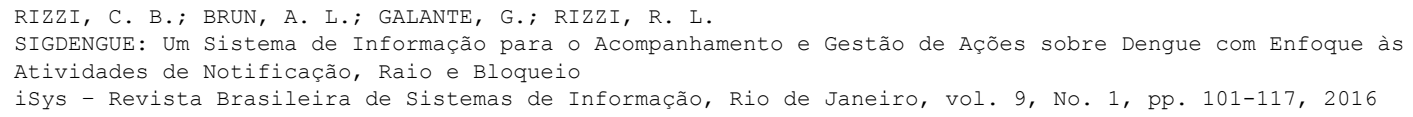


Mesmo considerando estes aspectos, melhorias pontuais foram sendo incorporadas diretamente no Sistema objetivando seu aperfeiçoamento e melhor adaptação ao trabalho realizado pelo setor responsável. Paralelamente novas funcionalidades foram identificadas e consideradas como fundamentais para comporem uma nova versão do Sistema. Duas delas merecem estaque: viabilizar que o SIGDENGUE pudesse ser utilizado por meio da Internet e que realizasse georreferenciamento.

A decisão de viabilizar a utilização por meio da Internet decorreu de diversos fatores, mas principalmente por que o mecanismo de funcionamento é utilizado pelos usuários já acostumados com navegadores web; atende às necessidades de usuários distribuídos remotamente; o desenvolvimento, manutenção e atualização ficam centralizados na aplicação sendo necessário apenas instalar ou alterar a aplicação no servidor para que os usuários possam acessá-la; a exportação de dados entre usuários remotos é viabilizada, inclusive por meio de aparelhos móveis; a escalabilidade no processamento permite que se aumente o poder de processamento apenas no servidor, caso necessário.

A decisão de dotar o SIGDENGUE com funcionalidade de georreferenciamento decorre principalmente do fato de que a análise espacial de fenômenos geográficos constitui uma forte aliada nas ações de entendimento, planejamento, gestão e enfrentamento de problemas. Permite, dentre outros aspectos, analisar a distribuição da doença e sua relação com o espaço geográfico onde ocorre. Assim, o SIGDENGUE poderia contribuir mais efetivamente para que o gestor pudesse tomar medidas cabíveis, específicas e em conformidade com o cenário em questão. Por exemplo, o mapeamento da distribuição da dengue viabiliza a identificação visual de áreas de risco e pontos estratégicos que merecem ação prioritária.

Principalmente por estes motivos, a equipe desenvolvedora do SIGDENGUE decidiu por reescrever seu código, convertendo-o em um Sistema de Informações Geográficas via Web. Iniciou-se então um trabalho em que os requisitos existentes foram revisados e outros foram elicitados. Incluiu-se um módulo para viabilizar a utilização de dispositivos móveis para coleta e transmissão de informações por agentes de campo. Um documento de requisitos foi elaborado e protótipos foram desenvolvidos e avaliados por representantes do Setor de Endemias do Município de Cascavel. Ajustes foram incorporados aos protótipos e as funcionalidades atualmente estão sendo implementadas.

Estão sendo utilizadas tecnologias que têm se mostrado interessantes do ponto de vista de que atendem à característica de funcionamento integrado via internet. HTML5, CSS, JavaScript, Bootstrap, Ajax e Java são utilizados na implementação. Para facilitar a modelagem e organização do projeto, optou-se pelo PlayFramework. Para o gerenciamento dos dados foi definido o SGBD PostGresSQL. Para a implementação do módulo de georreferenciamento, estão sendo utilizados o GEOSERVER e a biblioteca OPENLAYERS bem como arquivos do tipo shapefiles, fornecidos pela Prefeitura Municipal de Cascavel, e outros desenvolvidos no âmbito deste projeto.

RIZZI, C. B.; BRUN, A. L.; GALANTE, G.; RIZZI, R. L.

SIGDENGUE: Um Sistema de Informação para o Acompanhamento e Gestão de Ações sobre Dengue com Enfoque às Atividades de Notificação, Raio e Bloqueio

iSys - Revista Brasileira de Sistemas de Informação, Rio de Janeiro, vol. 9, No. 1, pp. 101-117, 2016 


\section{Referências}

Azevedo, L. H. A. (2007). A Geomática no contexto da ciência e tecnologia. Simpósio Brasileiro de Geomática. Presidente Prudente, 2007. Disponível em: http://docs.fct.unesp.br/departamentos/cartografia/ eventos/2007_II_SBG/artigos/A_012.pdf>. Acesso em Nov./2015.

Barros, D. M. d. S., Morais, P. S. G., Paiva, J. C., Lima, J. R. F., and Silva, J. L. R. (2013). Observatório nacional da dengue - sistema para monitoramente de casos de dengue. Revista Brasileira de Inovação Tecnológica em Saúde, 3(4):1-14.

Duncombe, J., Clements, A., Hu, W., Weinstein, P., Ritchie, S., and Espino, F. E. (2012). Geographical information systems for dengue surveillance. Am J Trop Med Hyg, 86(5):753-755.

Ecovec (2015). Disponível em: http: / / www . ecovec. com. Acesso em Set./2015.

Instituto de Saúde Coletiva - UFBA (2015). Dengue na Web. Disponível em: http: / / www. denguenaweb. org/index. php. Acesso em Nov./2015.

Lima, V. L., Andrade, V. R., Restitutti, M. C., Yanagizazawa J. S., N., and Carmo, R. L. (2004). The use of a geographic information system in dengue epidemiology, campinas-brazil, 2001-2003. Journal of Epidemiology \& Community Health, 58:103.

Martinez, R. (2006). Geographic information system for dengue prevention and control. Technical report, World Health Organization.

Ministério da Saúde (2010). SisPNCD. Sistema do Programa Nacional de Controle da Dengue. Manual do Usuário: Módulo Local. Disponível em: http: / / www . saude . rr.gov.br/visa/sispncd/manuais/sispncd_manuallocal.pdf.

Acesso em Set./2015.

Ministério da Saúde (2011). Portaria n. 104, de 25 de janeiro de 2011. Disponível em: http://bvsms . saude.gov.br/bvs/saudelegis/gm/2011/ prt0104_25_01_2011.html. Acesso em Set./2015.

Ministério da Saúde (2015a). Boletim Epidemiológico. V. 46 n. 31. Disponível em: http://portalsaude.saude.gov.br/images/pdf/2015/ outubro/15/svsbe-denchikzik-v46-n31.pdf. Acesso em Out./2015.

Ministério da Saúde (2015b). Levantamento Rápido do Índice de Infestação por Aedes Aegypti (LIRAa). Disponível em: http: / / www. dengue.org.br/dengue_ levantamento_municipios.pdf. Acesso em Set./2015.

Ministério da Saúde (2015c). Sistema de Informação de Agravos de Notificação - Sinan. Disponível em: http://dtr2004.saude.gov.br/sinanweb/. Acesso em Set./2015.

Mustafa, M., Rasotgi, V., Jain, S., and Gupta, V. (2015). Discovery of fifth serotype of dengue virus (denv-5): A new public health dilemma in dengue control. Medical Journal Armed Forces India, 71(1):67 - 70.

Palmer, S. and Felsing, J. (2002). A Practical Guide to Feature Driven Development. The Coad Series. 
Portal da Saúde (2015). Casos de Dengue. Brasil, Grandes Regiões e Unidades Federadas, 1990 a 2014. Disponível em: portalsaude.saude.gov.br/images/pdf/ 2015/julho/29/Dengue-at---2014.pdf. Acesso em Out./2015.

PROCC (2015). Info Dengue Rio - Situação da Dengue no Rio de Janeiro. Disponível em: http://www.procc.fiocruz.br/Members/claudia/ projeto-alerta-dengue. Acesso em Set./2015.

Pronex-Dengue (2015). Projeto Dengue. Disponível em: http://wiki . procc.fiocruz.br/mediawiki/index.php/Projeto_Dengue. Acesso em Set./2015.

Sesau-TO (2015). Sesau desenvolve sistema de informação de Dengue. Disponível em: http://secom.to.gov.br/noticia/41414/. Acesso em Set./2015.

TerraLab (2012). DengueME. Disponível em: http://www.decom.ufop.br/ terralab/. Acesso em Set./2015.

WHO (2006). World Health Organization. Dengue and severe dengue. Search Engine Strategies Conference 2006. Disponível em: http://www.who.int/ mediacentre/factsheets/fs117/en/. Acesso em Set./2015.

WHO (2009). Dengue: guidelines for diagnosis, treatment, prevention and control - new edition. Technical report, World Health Organization.

WHO (2015). DengueNet. Disponível em: http://apps.who.int/ globalatlas/default. asp. Acesso em Set./2015. 